\section{(6) OPEN ACCESS}

\title{
Transferring patients home to die: what is the potential population in UK critical care units?
}

\author{
Maureen A Coombs, ${ }^{1}$ Anne-Sophie E Darlington, ${ }^{2}$ Tracy Long-Sutehall, ${ }^{2}$ \\ Natalie Pattison, ${ }^{3}$ Alison Richardson ${ }^{2}$
}

\begin{abstract}
- Additional material is published online only. To view please visit the journal online (http://dx.doi.org/10.1136/ bmjspcare-2014-000834)

${ }^{1}$ Graduate School of Nursing, Midwifery and Health, Victoria University of Wellington, Wellington, New Zealand ${ }^{2}$ Faculty of Health Sciences, University of Southampton, Southampton, UK

${ }^{3}$ Royal Marsden NHS Foundation Trust, London, UK
\end{abstract}

\section{Correspondence to} Professor Maureen A Coombs, Graduate School of Nursing, Midwifery and Health, Victoria University of Wellington, Capital and Coast District Health Board, Wellington, 6021, New Zealand; maureen.coombs@vuw.ac.nz

Received 16 December 2014 Revised 2 September 2015 Accepted 12 November 2015 Published Online First 1 December 2015

\footnotetext{
To cite: Coombs MA, Darlington A-SE, LongSutehall T, et al. BMJ Supportive \& Palliative Care 2017:7:98-101.
}

\section{ABSTRACT}

Objectives Most people when asked, express a preference to die at home, but little is known about whether this is an option for critically ill patients. A retrospective cohort study was undertaken to describe the size and characteristics of the critical care population who could potentially be transferred home to die if they expressed such a wish.

Methods Medical notes of all patients who died in, or within 5 days of discharge from seven critical care units across two hospital sites over a 12-month period were reviewed. Inclusion/ exclusion criteria were developed and applied to identify the number of patients who had potential to be transferred home to die and demographic and clinical data (eg, conscious state, respiratory and cardiac support therapies) collected.

Results 7844 patients were admitted over a 12-month period. 422 (5.4\%) patients died. Using the criteria developed 100 (23.7\%) patients could have potentially been transferred home to die. Of these 41 (41\%) patients were diagnosed with respiratory disease. 53 (53\%) patients were conscious, 47 (47\%) patients were self-ventilating breathing room air/oxygen via a mask. $20(20 \%)$ patients were ventilated via an endotracheal tube. 76 (76\%) patients were not requiring inotropes/vasopressors. Mean time between discussion about treatment withdrawal and time of death was $36.4 \mathrm{~h}(\mathrm{SD}=46.48)$. No patients in this cohort were transferred home.

Conclusions A little over $20 \%$ of patients dying in critical care demonstrate potential to be transferred home to die. Staff should actively consider the practice of transferring home as an option for care at end of life for these patients.

\section{INTRODUCTION}

While most people indicate a preference to be cared for at home at the end of life, ${ }^{1}$ $58 \%$ of people in the UK die in hospitals. ${ }^{2}$
In critical care settings, the majority of deaths occur after planned treatment withdrawal and not following sudden patient deterioration. ${ }^{3}$ There is the possibility therefore, to consider patient and families wishes on preferred place of care at end of life. However, currently most patients and families would not be offered a choice about place of death and there are very few accounts in the literature of enabling the transfer of critically ill patients home to die. ${ }^{4}{ }^{5}$ A systematic search of Medline, CINAHL, Psychinfo and Embase databases identified only 13 papers published between 1980 and 2012 that discussed the transfer of adult patients from intensive care unit (ICU) home to die. The international practices reported on identified that the prevalence of transferring patients home to die is low, it is often informed by cultural preferences about the place of death, and driven by patient/family choice in informing the decision to transfer home.

To date, the work in this area ${ }^{6}$ offers little in the way of detail as to which type of patient, or indeed how many patients, could be considered for transfer home as part of end of life care. With international health policy focusing on the provision of high quality end of life care informed by patient choice about place of care, ${ }^{8}$ the profile of this critical care population merits further study.

\section{AIM}

To determine the size and characteristics of the critical care population who could potentially be transferred home to die.

\section{METHODS}

A retrospective 12 month cohort study was undertaken across four ICU settings 
(general, cardiac, neurological and oncology) and three high dependency unit (HDU) settings (Medical, Cardiac and Surgical) in two hospital sites in the South of England.

The medical notes of all patients admitted to the ICU/HDUs during 1 January 2011 to 31 December 2011 and who subsequently died in the ICU/HDU or within 5 days of discharge from these units, were reviewed. Most patient dying within 5 days of discharge from ICU would have been identified as patients for supportive treatment only, and therefore had potential for a transfer home to die.

To determine potential size of the population who could potentially be transferred home to die, the characteristics of this population needed to be ascertained. These were developed from previous focus group work with ICU doctors and nurses where clinical and social circumstances mitigating for and against transfer of critically ill patients home to die were identified. ${ }^{9}$

From this, patients were excluded from the audit and deemed unsuitable for transfer if there was documented evidence of: sudden death (cardiac arrest or other cause); patient instability in the $24 \mathrm{~h}$ preceding planned treatment withdrawal (based on significant deterioration, introduction of new intensive treatments, large inotrope/ vasopressor requirements, or numerous changes in interventions); involvement of the coroner or police in the case notes; complex family dynamics; or physically demanding care needs (unstable spine, morbid obesity, large gastrointestinal losses, complex wound care).

A data collection tool was developed (and reviewed within the study team) to collect information on patients who had died, those included and excluded from the study (Appendix A). The final version was pilot-tested with 10 medical notes from one ICU. An Intensivist (not on the research team) independently reviewed these 10 sets of notes and completed the data collection tool as a further quality check.

For patients included in the study, specific data were collected on: respiratory status and respiratory interventions; cardiovascular support drugs (specifically inotropic/vasopressors); and state of consciousness (defined as conscious or not). Demographic data were also collected including: patient age; gender; type of unit; level of care (as assessed by UK Department of Health: Comprehensive Critical Care criteria); ${ }^{10}$ diagnosis; date and time of discussion of withdrawal with family, and date and time of death.

Data collection was undertaken by ICU research nurses based in the two hospital sites. All had a clinical background in critical care. Any data collection issues were discussed with the Intensivist and experienced clinicians on the research team until a consensus decision was reached.

\section{DATA ANALYSIS}

Data were imported into SPSS with descriptive and inferential statistics used for analysis. Comparisons between the patients eligible for transfer and patients not eligible for transfer were carried out investigating age and unit type (HDU vs intensive therapy unit (ITU)). Difference in age was investigated using a Mann-Whitney $\mathrm{U}$ test, as age data were not normally distributed (Shapiro-Wilk $\mathrm{p}=0.000$ ). Differences according to unit type were investigated using a $\chi^{2}$ test. All patient data used in this study were handled and processed in accordance with National Health Service (NHS) best practice ${ }^{11}$ and Caldicott recommendations. ${ }^{12}$ Site specific research governance procedures were adhered to.

\section{RESULTS}

A total of 7844 patient records from four ICU and three HDU settings were reviewed: general ICU $(n=1548)$, cardiac ICU $(n=1298)$, neurological ICU $(n=738)$, oncology ICU $(n=1313)$, surgical HDU $(n=869)$, cardiac HDU $(n=1717)$ and medical HDU $(n=361)$. Of these, $422(5.4 \%)$ patients had died in the ICU/HDU or within 5 days of discharge from these units. None were transferred home to die.

Of the 422 deceased patients, 322 (76.3\%) were excluded as ineligible for transfer (see also figure 1). Sixty-two $(14.7 \%)$ patients were excluded due to sudden death (cardiac or respiratory arrest). A further $225(53.3 \%)$ patients were excluded due to physiological/clinical instability and $35(8.3 \%)$ patients on the basis of documented family/legal issues related to the patient or the need for physically demanding care needs which would prove difficult in a home environment (eg, profuse diarrhoea or vomiting, complex wound care).

There were $100(23.7 \%)$ patients who, based on the developed criteria, could have potentially been

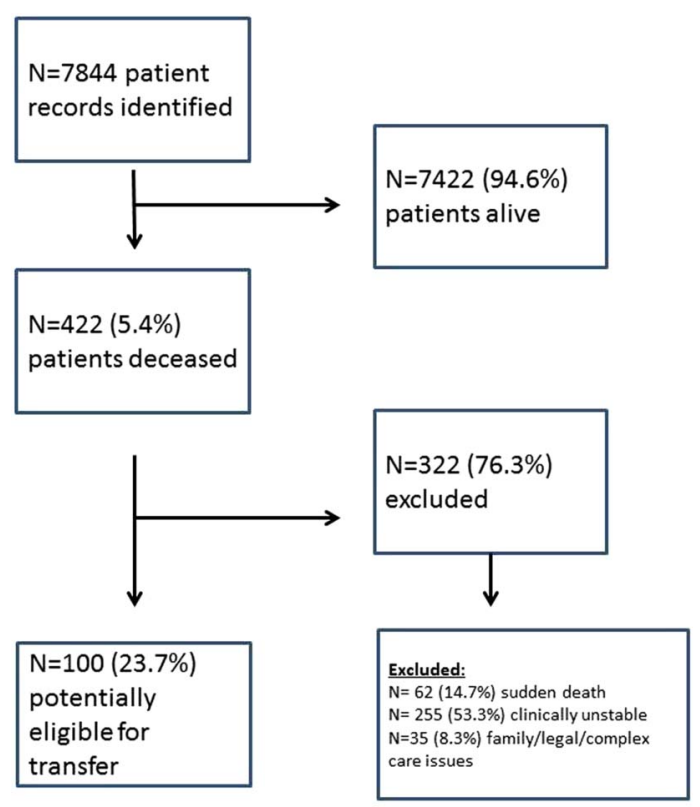

Figure 1 Flowchart of patients. 
considered for transfer home to die if this had been offered/requested. Of the 100 patients identified as eligible for transfer, $44(44 \%)$ patients were female and $56(56 \%)$ male. The average age was 70.4 years $(\mathrm{SD}=13.03$, range $23-92$ years).

$31(31 \%)$ patients were recorded as requiring level 1 care (acute care ward with support from critical care), 45 (45\%) patients level 2 (eg, HDU) and 24 (24\%) patients level 3 (eg, ICU). The majority of patients had been diagnosed with respiratory $(n=41$, $41 \%)$, neurological $(n=19,19 \%)$ or cardiac disease $(n=19,19 \%)$. The mean time between discussion about withdrawal of treatment during a family meeting and time of death was $36.36 \mathrm{~h}(\mathrm{SD}=46.48$; see table 1$)$.

Fifty-three $(53 \%)$ patients from the potential pool eligible for transfer were conscious. Thirteen (13\%) of the group were self-ventilating breathing room air with no support, 34 (34\%) were self-ventilating with oxygen via a mask and $18(18 \%)$ were receiving noninvasive ventilation via a mask. Twenty (20\%) of patients were ventilated via an endotracheal tube.

With regards to cardiovascular support, 76 (76\%) patients were not receiving any inotropes or vasopressors, $8(8 \%)$ patients were receiving a single inotrope or vasopressor (excluding 'low dose' dopamine), 3 (3\%) patients were only receiving dopamine. Only 4 (4\%) of the identified potential transfer home to die group were receiving multiple inotropes/vasopressors (information was missing for 8 cases).

No statistically significant difference was found in terms of age (Mann-Whitney $\mathrm{U}=17475, \mathrm{p}=0.15$ ) or patient sex $\left(\chi^{2}=0.001, p=0.53\right)$ for patients who were included for potential transfer and those who were excluded from potential transfer. Patients who were eligible for transfer were statistically significantly more likely to be treated in HDU than ITU compared to patients who were excluded $\left(\chi^{2}=19.80, \mathrm{p}<0.001\right)$ : $18.4 \%(n=58$ of 316$)$ of the ICU patient sample would have been eligible for transfer versus $39.6 \%$ $(n=42$ of 106$)$ of the HDU sample of patients who had died. Figure 2 illustrates the differences between the different units in terms of the proportion of patients, from the group of 422 deceased patients, not eligible for transfer (excluded) versus the proportion of patients potentially eligible for transfer (include): (exclude vs include) cardiac HDU $7.1 \%$ vs $14 \%$; cardiac ICU $13.3 \%$ vs $3 \%$; general ICU $45 \%$ vs $31 \%$; medical HDU 9.9\% vs 26\%; neurological ICU $8.4 \%$ vs $17 \%$; oncology ICU $13.4 \%$ vs $7 \%$; surgical HDU $2.9 \%$ vs $2 \%$.

\section{DISCUSSION}

The aim of the study was to determine the size and characteristics of the critical care population who could potentially be transferred home to die, based on a set of predetermined criteria about clinical parameters. Approximately $20 \%$ of patients who died in
Table 1 Characteristics of patients potentially eligible for transfer

Patient characteristics

Patients eligible for transfer, $\mathrm{n}(\%)$

$100(23.7)$

Patient sex, female $\mathrm{n}(\%)$

$44(44)$

Patient age, mean (SD), range

70.4 years

$(\mathrm{SD}=13.03)$

23-92 years

The mean time between discussion about withdrawal 36.36 (46.48) of treatment during a family meeting and time of death, mean (SD) hours

Level of care

Level 1 care (acute care ward with support from critical care), $\mathrm{n}(\%)$

Level 2 care (eg, HDU), n (\%)

$31(31)$

Level 3 care (eg, ICU), n (\%)

Disease category (for majority of patients)

Respiratory, n (\%)

$41(41)$

Neurological, $n(\%)$

19 (19)

Cardiac disease, n (\%)

19 (19)

Respiratory support

Self-ventilating breathing room air with no

support

Receiving non-invasive ventilation via a mask 18 (18)

Ventilated via an endotracheal tube

$20(20)$

Self-ventilating with oxygen via a mask

State (conscious/unconscious)

Conscious, $\mathrm{n}(\%)$

Cardiovascular support

Dopamine only, n (\%)

Receiving multiple inotropes/vasopressors, $\mathrm{n}(\%)$

Receiving a single inotrope or vasopressor

(excluding 'low dose' dopamine), n (\%)

Not receiving any inotropes or vasopressors, $\mathrm{n}(\%) \quad 76(76)$

Data does not always add up to $100 \%$ due to missing data for a small number of patients.

ICU and HDU environments in our sample met these criteria and could therefore potentially be considered for transfer home to die, should this be their wish. These patients are likely to be those who are breathing

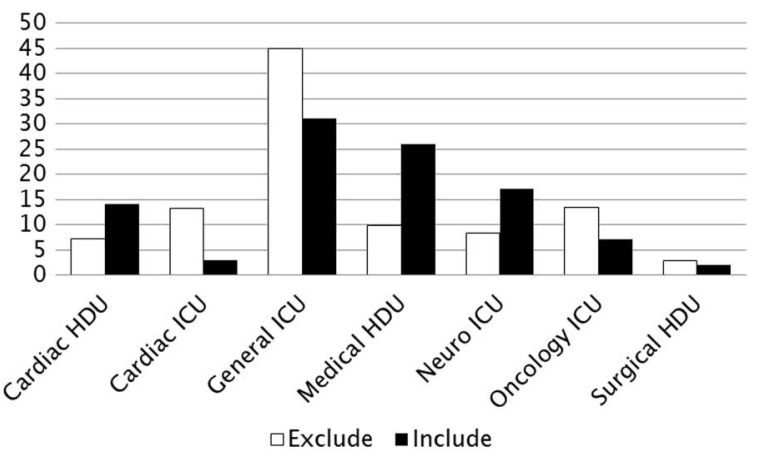

Figure 2 Potential patients: type of unit/specialty (expressed in percentages). ICU, intensive care unit; HDU, high dependency unit. 
spontaneously, not requiring cardiovascular support and not having complex care needs.

This is the first large scale study to quantify this particular critical care population. In doing this, it takes the reporting in this area beyond case reports ${ }^{13} 14$ and provides more detailed information on the types of patients for whom transfer home may be an option. This may help inform the debate about how patient wishes at end of life might be addressed in critical care. $^{15}$

This study has only explored physiological and care delivery indicators to determine size and characteristics of this group. Individual preferences, cultural and spiritual factors and availability of healthcare resource can significantly influence decisions about transferring patients' home to die. These factors need further consideration. The data collection tool was developed for the adult population. Any replication studies outside this field for example, neonates, paediatrics will require refinement of the data collection tool.

\section{CONCLUSION}

Based on physiological criteria and care requirements, approximately $20 \%$ of critical care patients where treatment withdrawal is being considered have potential to be considered for transfer home at end of life. While this remains a small subset of the critically ill patient population, critical care doctors and nurses can use these characteristics to actively consider whether transfer home may be a component of end of life care for some patients.

Correction notice This article has been corrected since it published Online First. The Open Access licence has been added.

Acknowledgements The authors would like to thank Dr Kathleen Nolan, Kim Golder, Clare Bolger and Geraldine O'Gara for the work they undertook in relation to the study.

Contributors MAC contributed substantially to the conception and design of the work, and the acquisition, of the data as well as the analysis and interpretation of data. MAC contributed to drafting and revising the manuscript as well as giving final approval of the version submitted. A-SED contributed substantially to the conception and design of the work, and the acquisition, of the data as well as the analysis and interpretation of data. A-SED contributed to drafting and revising the manuscript as well as giving final approval of the version submitted. A-SED submitted the manuscript. TL-S contributed substantially to the conception and design of the work, and the acquisition, of the data as well as the analysis and interpretation of data. TL-S contributed to drafting and revising the manuscript as well as giving final approval of the version submitted. NP contributed substantially to the conception and design of the work, and the acquisition, of the data as well as the analysis and interpretation of data. NP contributed to drafting and revising the manuscript as well as giving final approval of the version submitted. AR contributed substantially to the conception and design of the work, and the acquisition, of the data as well as the analysis and interpretation of data.
AR contributed to drafting and revising the manuscript as well as giving final approval of the version submitted.

Funding This work was part of a mixed methods study funded by Marie Curie Cancer Care.

Competing interests None declared.

Provenance and peer review Not commissioned; externally peer reviewed.

Open Access This is an Open Access article distributed in accordance with the Creative Commons Attribution Non Commercial (CC BY-NC 4.0) license, which permits others to distribute, remix, adapt, build upon this work noncommercially, and license their derivative works on different terms, provided the original work is properly cited and the use is non-commercial. See: http://creativecommons.org/licenses/by$\mathrm{nc} / 4.0 /$

\section{REFERENCES}

1 Gomes G, Calanzani N, Gysels M, et al. Heterogeneity and changes in preferences for dying at home: a systematic review. BMC Palliat Care 2013;12:7.

2 National End of Life Care Intelligence Network. Variations in Place of Death in England: inequalities or appropriate consequences of age, gender and cause of death. National End of Life Care Intelligence Network, 2010.

3 Frick S, Uehlinger D, Zuercher Zenklusen R. Medical futility: predicting outcome of intensive care patients by nurses and doctors-a prospective comparative study. Crit Care Med 2003;31:456-61.

4 Huang Y, Huang S, Ko W. Going home to die from surgical intensive care units. Intensive Care Med 2009;35:810-15.

5 Mann S, Galler D, Williams P, et al. Caring for patients and families at the end of life: withdrawal of intensive care in the patient's home. N Z Med J 2004;117:935-43.

6 Kallel H, Dammak H, Bahloul M, et al. A good death: another break in the wall. Intensive Care Med 2006;32:1915-16.

7 Boussarsar M, Bouchoucha S. Dying at home: cultural and religious preferences. Intensive Care Med 2006;32:1917-18.

8 Department of Health. One change to get it right. Improving people's experience of care in the last few days and hours of life. London: Leadership Alliance for the Care of Dying People, Department of Health, 2014.

9 Coombs M, Long-Sutehall T, Darlington AS, et al. Doctors' and nurses' views and experience of transferring patients from critical care home to die: a qualitative exploratory study. Palliat Med 2015;29:354-62.

10 Department of Health. Comprehensive critical care: a review of adult critical care services. London: Department of Health, 2000.

11 NHS Confidentiality Code of Practice 2003. http://www.dh. gov.uk/en/Policyandguidance/Informationpolicy/ Patientconfidentialityandcaldicottguardians/DH_4100550

12 Caldicott Committee Report 1997. http://www.dh.gov.uk/en/ Publicationsandstatistics/Publications/PublicationsPolicy AndGuidance/DH_4068403

13 Lusardi P, Jodka P, Stambovsky M, et al. The going home initiative: getting critical care patients home with hospice. Crit Care Nurse 2011;31:46-57.

14 Tellett L, Davis C. Case 43 fulfilling a patient's wish to go home from intensive care. Eur J Palliat Care 2009;16:69-70.

15 Department of Health. More care, less pathway. A review of the Liverpool Care Pathway. London: Department of Health, 2013. 